\title{
NK-pDC cross-talk leads to the generation of mTRAIL+IFN- $\alpha+$ cytotoxic pDCs following HIV-1 infection of pDCs. Consequences on the control of viral replication
}

Héla Saïdi, Marlene Bras, Formaglio Pauline, Melki Marie-thérèse, Gougeon Marie-Lise

From $16^{\text {th }}$ International Symposium on HIV and Emerging Infectious Diseases

Marseille, France. 24-26 March 2010

\section{Background}

Plasmacytoïd dendritic cells (pDCs) play a central role in orchestrating innate and adaptive immunity, especially by secreting large amounts of IFN- $\alpha$ in response to viral stimuli. IFN- $\alpha$ production by $\mathrm{pDCs}$ depends upon a cross-talk with NK cells that triggers the activation of NK cells. Considering that NK cells are essential for the elimination of virus-infected cells, and pDC required for their antiviral activity, we addressed the impact of HIV on NK-pDC cross-talk, and the consequences on viral control.

\section{Methods}

pDCs and NK cells were sorted with magnetic beads from the blood of healthy donors. NK cells were kept either resting or activated with PMA/ionomycine for 2 hrs. pDC were stimulated with either IL-3 or CpG, or were infected with R5-HIV-1 at various concentrations. pDCs were cocultured with NK cells at various ratios for $24 \mathrm{hrs}$. The influence of NK-pDC interaction on both cell types was analyzed by multiparametric flow cytometry, combining maturation and cell death/survival markers with cytokine detection, and released cytokines in cultures supernatants were identified and quantified by the MAP luminex technique.

\section{Results}

Our results show that high amounts of HIV-1 induced the maturation of pDCs, characterized by the expression of HLA-DR, CD40, CD80, CD86, CCR7 and CD83. This phenotypic maturation was coupled to a functional maturation since HIV-1-infected pDCs were able to activate the production of IFN- $\gamma$ and TNF- $\alpha$ by NK cells after 24 hrs of co-culture. Consequently NK cells induced, in synergy with the virus, the emergence of killer mTRAIL+IFN- $\alpha+$ pDCs. In contrast, NK-pDC cross-talk did not induce pDC maturation at low concentration of HIV, and it had a poor effect on the activation of NK cells. Finally, the release of $\beta$-chemokines and IFN- $\alpha$ was found dependent both on NK-pDC cross-talk and HIV concentration.

\section{Discussion}

We report for the first time that NK-pDC cross-talk induces the maturation and differentiation pDCs into mTRAIL+ IFN- $\alpha+$ cytotoxic pDCs once infected with HIV-1. Furthermore, depending on virus concentration, NK-pDC was found involved in the viral control, either by triggering or by suppressing the release of anti-HIV molecules, such as $\beta$-chemokines and IFN- $\alpha$.

Published: 11 May 2010

doi:10.1186/1742-4690-7-S1-04

Cite this article as: Saïdi et al:: NK-pDC cross-talk leads to the generation of mTRAIL+IFN- $\alpha+$ cytotoxic pDCs following HIV-1 infection of $\mathrm{pDCs}$. Consequences on the control of viral replication. Retrovirology 2010 7(Suppl 1):04.

\footnotetext{
* Correspondence: marlene.bras@pasteur.fr
}

Institut Pasteur, Paris, France 